\title{
Evaluación agronómica de líneas de arroz de sabana (Oryza sativa L.) obtenidas mediante mejoramiento poblacional por selección recurrente
}

\section{Agronomic evaluation of lines of sabana rice (Oryza sativa L.) obtained by population improvement with recurrent selection}

\author{
Hernando Delgado-Huertas ${ }^{1}$; Amanda Silva-Parra ${ }^{2}$; Luis Alfonso Guarín-Gutierrez ${ }^{3}$
}

1'Ing. Agrónomo, M.Sc. Universidad de los Llanos, Grupo de Investigación ISAF. Villavicencio - Meta, Colombia; e-mail: hdelgado@unillanos.edu.co; (D) https://orcid. org/0000-0003-2627-7174

${ }^{2}$ Ing. Agrónomo, Ph.D. Universidad de los Llanos. Villavicencio - Meta, Colombia; e-mail: asilvap@unillanos.edu.co; (D) https://orcid.org/0000-0001-9872-790X

${ }^{3}$ Ing. Agrónomo, M.Sc. Universidad de los Llanos. Villavicencio - Meta, Colombia; e-mail: Iguarin@unillanos.edu.co; (D) https://orcid.org/0000-0001-7924-4726

Cómo citar: Delgado-Huertas, H.; Silva-Parra, A.; Guarín-Gutierrez, L.A. 2021. Evaluación agronómica de líneas de arroz de sabana (Oryza sativa L.) obtenidas mediante mejoramiento poblacional por selección recurrente. Rev. U.D.C.A Act. \& Div. Cient. 24(2):e1707. http://doi.org/10.31910/rudca.v24.n2.2021.1707

Artículo de acceso abierto publicado por Revista U.D.C.A Actualidad \& Divulgación Científica, bajo una Licencia Creative Commons CC BY-NC 4.0

Publicación oficial de la Universidad de Ciencias Aplicadas y Ambientales U.D.C.A, Institución de Educación Superior Acreditada de Alta Calidad por el Ministerio de Educación Nacional.

Recibido: septiembre 7 de $2020 \quad$ Aceptado: junio 25 de 2021

Editado por: Ingeborg Zenner de Polanía

\section{RESUMEN}

Los métodos clásicos de mejoramiento han estrechado la base genética del arroz. La selección recurrente (SR) permite el mejoramiento de una población, mediante la pirámidación de alelos favorables de diversos donantes y de origen genético diverso. Ha sido usada en Colombia por CIAT/CIRAD, para el desarrollo y el mejoramiento del acervo genético del arroz Oryza sativa L., tipo Japonica Tropical de secano en sabanas ácidas. Se evaluaron en bloques completos al azar, con tres repeticiones, 50 líneas avanzadas junto con el testigo comercial Corpoica Llanura 11. Se observó diferencia significativa entre líneas de arroz para las variables dependientes rendimiento de grano, días a floración, vigor e incidencia de Pyricularia en hojas y cuello, así como manchado de grano. Las líneas 39 y 37, de la población PCT-11, presentaron los mayores rendimientos, con 6,471 y 6,192 $\mathrm{kg} \mathrm{ha}^{-1}$, respectivamente, diferentes significativamente del testigo y de nueve líneas de ciclo tardío de la población PCT-4, que presentaron rendimientos muy bajos. Especialmente, la línea 39, mostró menor incidencia del hongo Pyricularia en hojas, que el testigo comercial Corpoica Llanura 11. Los presentes resultados muestran la utilidad de la SR en arroz de sabanas, para generar líneas superiores en rendimiento, en resistencia a enfermedades, en adaptación a las sabanas ácidas y el potencial para contribuir a la diversificación de su base genética.

Palabras clave: Genotipo; Ambiente; Adaptación; Rendimiento; Sanidad. 


\section{ABSTRACT}

The classical breeding methods have narrowed the genetic base of rice. The pedigree method with a limited number of elite and genetically related parents, can reduce variability in long term studies. The development of populations with a broad genetic base and the use of breeding methods that allow the continuous accumulation of favorable alleles could overcome these disadvantages. Recurrent selection (SR) allows the improvement of a population through the pyramidation of favorable alleles of diverse donors and of diverse genetic origin. It has been used in Colombia by CIAT/ CIRAD for the development and improvement of the genetic stock of Oryza sativa L. rice, a tropical rain-fed Japonica type in acid savannas. They were evaluated in complete blocks at random with three repetitions, 50 advanced lines of this project, together with the commercial control Corpoica Llanura 11. There was a significant difference between rice lines for the dependent variables grain yield, days to flowering, vigor and incidence of Pyricularia in leaves and spotted of grain. Lines 39 and 37 of the PCT-11 population presented the highest yields, with 6.471 and $6.192 \mathrm{~kg}$ $\mathrm{ha}^{-1}$, respectively, significantly different from the control and nine late cycle lines of the PCT-4 population, which presented very low yields. Especially line 39 showed a lower incidence of the Pyricularia fungus on leaves than the commercial control Corpoica Llanura 11. The present results and others obtained in Colombia and Brazil, show the utility of SR in savanna rice to generate superior lines in yield, resistance to diseases, adaptation to acid savannas and the potential to contribute to the diversification of the genetic base.

Keywords: Genotype; Environment; Adaptation; Yield; Plant health.

\section{INTRODUCCIÓN}

Los métodos clásicos de mejoramiento han conducido a que se estreche la base genética del arroz (Oryza sativa L.) (Cuevas-Perez et al. 1992; Rangel et al. 1996; Montalvan et al. 1998). Métodos, como el de pedigrí, generalmente, involucran en su cruce un número limitado de progenitores élite, con frecuencia relacionados genéticamente y con riesgo a largo plazo de reducción de la variabilidad. El desarrollo de poblaciones con amplias bases genéticas y el uso de métodos de mejoramiento, que permitan la acumulación continua de alelos favorables, podrían superar estas desventajas. La selección recurrente (SR), se puede ajustar al mejoramiento de una población, mediante la eficiente pirámidación de diferentes alelos favorables, de diversos donantes (Xu \& Ali, 2014; Gazal et al. 2016; Herawati et al. 2017b).

Se han desarrollado varios grupos de genes y de poblaciones, que se han utilizado como base genética para la SR, en diferentes países de América Latina, como Brasil, Chile y Colombia y, en varios países africanos, como Costa de Marfil, Mal y Madagascar (Das, 2018).

La creación de poblaciones con amplia base genética y su mejoramiento a través de SR, ha sido usada desde 1996, en Colombia, por CIAT/CIRAD, para el desarrollo y el mejoramiento del acervo genético del arroz, tipo Japonica Tropical, de tierras altas o de secano en sabanas ácidas. Mediante el uso de un gen recesivo de esterilidad masculina ( $\mathrm{ms}$ ), de un mutante de IR36 (Singh \& Ikehashi, 1981), se facilitó el desarrollo y la mejora de las poblaciones de arroz. El programa comenzó con el desarrollo de una población base, a través del cruce de, aproximadamente, 60 accesiones fundadoras complementarias. Dicha población, se recombinó varias veces, para generar un gran número de recombinantes, lo que condujo a una población sintética de desequilibrio de ligamiento bastante bajo, dotado de suficiente variabilidad genética, para la selección futura (Grenier et al. 2015). Plantas fértiles, se seleccionan de las poblaciones compuestas básicas en cada paso de mejoramiento por SR y son el punto de partida para la selección de progenies segregantes, por el método de pedigrí convencional y la identificación de líneas fijas promisorias (Chatel \& Guimaraes, 2000; Chatel et al. 2008; Chatel et al. 2010). Sì se explora la amplia variabilidad genética presente en esas poblaciones, resultarán variedades de base genética distinta de las que están hoy disponibles en el mercado latinoamericano (Chatel et al. 2010).

El mejoramiento de poblaciones por SR es eficiente para mejorar características cuantitativas y de baja heredabilidad. Además, permite romper los bloques de ligamiento génico que, en el arroz, se constituyeron durante muchos años de selecciónautofecundación y liberar variabilidad genética, mediante ciclos sucesivos de cruzamientos, o sea, por las recombinaciones (Chatel et al. 2010). En otras palabras, este método es un procedimiento de recopilación de los caracteres deseados de una combinación de cruces con algunos cruces continuos de segregantes seleccionados, para obtener una mejor población que la anterior. Se ha utilizado en especies de plantas para mejorar el rendimiento, alterar la calidad de las semillas o de las plantas, aumentar la resistencia a las plagas y mejorar la tolerancia al medio ambiente (Abdullah, 2009; Hallauer et al. 2010; Berilli et al. 2013; Morais Junior et al. 2015; Gazal et al. 2016; Herawati et al. 2017a; Herawati et al. 2017b).

Actualmente, en China, se cuenta con otra nueva fuente de esterilidad masculina, en este caso, dominante, introducida en el fondo genético del cultivar de arroz "Jiabuyu", que también puede ser usada en el mejoramiento por SR en arroz, haciendo más eficiente la recombinación, en comparación con la androesteterilidad recesiva usada anteriormente y, en Colombia, por CIAT/CIRAD, dado que las plantas androestériles pueden estar en una frecuencia de $50 \%$, en la primera generación. Además, la planta androfértil es homocigota, por lo que se pueden usar directamente como materiales de mejora, para la detección de rasgos. La población de SR desarrollada, a través de un solo material androestéril dominante, tiene las características ventajosas de cultivos de polinización cruzada y cultivos autopolinizados (Pang et al. 2017). También, Ansari et al. (2017), mediante silenciamiento génico por RNAi y transformación mediada por Agrobacterium, obtuvieron plantas enanas androestériles, para utilizar en SR en arroz.

Las variedades mejoradas de los denominados arroces de sabana en Colombia (Oryzica Sabana 6, Oryzica Sabana 10 
y CORPOICA Lanura 11), han sido obtenidas, hasta ahora, mediante mejoramiento convencional y selección por pedigrí, por el trabajo colaborativo de ICA, CORPOICA (hoy Agrosavia) y CIAT/CIRAD, en un programa de mejoramiento específico de adaptación a la alta acidez y concentración de $\mathrm{Al}$ intercambiable de las sabanas bien drenadas del piedemonte y de la altillanura plana del Meta, pues, a este ecosistema, no se adaptaban las variedades mejoradas obtenidas para los sistemas de riego por inundación y de secano favorecido en suelos aluviales; provienen de cruzamientos de progenitores africanos, de Brasil, México y Colombia. Son variedades que toleran la acidez y la toxicidad por aluminio de las sabanas, requiriendo cal dolomita, en dosis bajas de $300 \mathrm{~kg} \mathrm{ha}^{-1}$; su tipo de planta es de porte más alto que las variedades para riego, con tallos flexibles resistentes al volcamiento; ciclos precoces de 85 a 110 días, adecuados para rotaciones; un sistema radical grueso y profundo, adaptado al ecosistema de secano en sabanas; resistencia al hongo Pyricularia grisea y adecuada calidad molinera y culinaria. Se han utilizado en el sistema arroz-pastos para establecer pasturas mejoradas, en sistemas rotacionales con soya y maíz y en sistemas agroforestales, como cultivo intercalado en los primeros años de establecimiento de la especie perenne (Delgado \& Aristizábal, 2002; Delgado et al. 2004).

El objetivo del presente trabajo fue evaluar la adaptación agronómica preliminar de 50 líneas avanzadas de arroz de sabana, provenientes del proyecto de mejoramiento genético poblacional por SR de CIAT/CIRAD, a las condiciones de un suelo de sabana ácida, en terraza alta de Villavicencio.

\section{MATERIALES Y MÉTODOS}

Localización. El experimento, se sembró en el segundo semestre, el 24 de agosto de 2017, en un suelo de sabana ácida, de la Granja Barcelona, de la Universidad de los Llanos en Villavicencio, con coordenadas $4^{\circ} 04^{\prime} 47^{\prime \prime} \mathrm{N}$ y $73^{\circ} 17^{\prime} 35^{\prime \prime}$ O, altitud de $423 \mathrm{~m}$ s.n.m., con $27{ }^{\circ} \mathrm{C}$ de temperatura promedio anual, $85 \%$ de humedad relativa y una precipitación promedio anual de $3.500 \mathrm{~mm}$, con un régimen bimodal de lluvias.

Material vegetal. Se realizó evaluación agronómica de 50 líneas avanzadas del proyecto de selección recurrente para secano en sabanas ácidas, proporcionadas por CIRAD/CIAT (Palmira) y el testigo comercial Corpoica Llanura 11, también conocido como Línea 30 o CIRAD 409 (Tabla 1), una variedad mejorada para sabanas ácidas, obtenida por cruzamientos y selección pedigrí convencionales (Delgado et al. 2004).

Diseño experimental. Bloques completos al azar, con tres repeticiones. Unidad experimental parcela de 3 surcos, sembrados a chorrillo manual, cada uno de $3 \mathrm{~m}$ de longitud y $0,2 \mathrm{~m}$ de distancia entre surcos, para un área experimental por línea de $2,4 \mathrm{~m}^{2}$.

Manejo agronómico. Control químico de malezas, se aplicó Tiobencarbo en preemergencia $\left(4,0 \mathrm{Lha}^{-1}\right)$; postemergente temprano (Propanil 4,0 + Tiobencarbo 2,5 Lha ${ }^{-1}$ ). Fertilización: se aplicó fertilización equivalente a $100 \mathrm{~kg}$ de $\mathrm{P}_{2} \mathrm{O}_{5}, 100 \mathrm{~kg}$ de $\mathrm{K}_{2} \mathrm{O}$ y
$100 \mathrm{~kg}$ de $\mathrm{N} \mathrm{ha}^{-1}$. El P y la mitad del K, a la siembra al fondo del surco, la otra mitad del K con la primera aplicación de $\mathrm{N}$. El N en banda, fraccionado en tres aplicaciones (al inicio del macollamiento, al máximo del mismo y al inicio del primordio floral). Las dosis, se calcularon según niveles críticos de nutrientes en análisis químico del suelo.

Variables evaluadas. Con base en las escalas del sistema de evaluación estándar para arroz (Rosero, 1983), se evaluaron las características agronómicas: vigor, días a floración y rendimiento y el daño por las enfermedades más limitantes en la zona: Piricularia en la hoja (Pyricularia grisea), Helmintosporiosis (Helminthosporium oryzae), Escaldado de la hoja (Rhynchosporium oryzae), Manchado del Grano (Complejo de hongos) y Piricularia en el Cuello de la Panícula (Pyricularia grisea).

Análisis estadístico. Los datos obtenidos de todas las variables evaluadas fueron sometidos, mediante el programa INFOSTAT, a análisis de varianza y se realizaron pruebas de comparación de medias de Tukey, para las variables que mostraron diferencias estadísticas en el Anava.

\section{RESULTADOS Y DISCUSIÓN}

En el análisis de varianza (Tabla 2), se observó diferencia significativa entre líneas de arroz $(\mathrm{p}<0,01)$, para las variables dependientes rendimiento de grano (REND), días a floración (FLO), vigor y manchado de grano (MGRA). La incidencia del patógeno Pyricularia en hojas presentó diferencia significativa entre líneas $(\mathrm{p}<0,05)$.

La incidencia de Pyricularia en cuello de panículas (PC), Helminthosporiosis (HELM) y escaldado de las hojas (ESC) por Rhynchosporium oryzae, fue baja y sin diferencia significativa entre las líneas evaluadas (Tabla 3 ).

Seis líneas (identificadas con los números de orden 39, 37, 34, 48,36 y 23) y provenientes de la población PCT-11, presentaron los mayores rendimientos de grano, superiores a $6.000 \mathrm{~kg} \mathrm{ha}^{-1}$. El mayor rendimiento lo presentó la línea 39 , con $6,471 \mathrm{~kg} \mathrm{ha}^{-1}$, diferente significativamente de la variedad testigo Corpoica Llanura $11\left(2.510 \mathrm{~kg} \mathrm{ha}^{-1}\right)$ y de 9 líneas de ciclo tardío, todas ellas originadas en la población PCT-4, que presentaron rendimientos muy bajos, mostrando su baja adaptación agronómica al ambiente de sabanas ácidas; sin embargo, una línea de la población PCT-4, la número 22, con rendimiento de $4.751 \mathrm{~kg} \mathrm{ha}^{-1}$, sin diferencia significativa de la mejor línea, presentó un adecuado comportamiento. El rendimiento de la variedad testigo pudo estar afectado por bajo vigor germinativo de su semilla (Tabla 3).

Chatel et al. (2008) reportaron también que una línea extraída del primer ciclo de selección recurrente de la población PCT-4, rindió un $35 \%$ más y mostró la misma precocidad y buena adaptación que el mejor testigo comercial, la Línea 30, desarrollada por cruces convencionales. 
Tabla 1. Listado de líneas avanzadas de arroz de sabanas evaluadas. Villavicencio. 2017 B.

\begin{tabular}{|c|c|}
\hline $\begin{array}{l}\mathrm{N}^{\circ} \text { de } \\
\text { línea }\end{array}$ & Designación \\
\hline 1 & РCT - $4 \backslash \mathrm{SA} \backslash 1 \backslash 1, \mathrm{Bo} \backslash 6 \backslash 1>16-5-3-2-\mathrm{M}$ \\
\hline 2 & PCT $-4 \backslash \mathrm{SA} \backslash 1 \backslash 1, \mathrm{Bo} \backslash 6 \backslash 1>17-1-1-1-\mathrm{M}$ \\
\hline 3 & PCT - $4 \backslash \mathrm{SA} \backslash 1 \backslash 1, \mathrm{Bo} \backslash 6 \backslash 1>17-1-2-1-\mathrm{M}$ \\
\hline 4 & PCT - $4 \backslash \mathrm{SA} \backslash 1 \backslash 1, \mathrm{Bo} \quad \backslash 6 \backslash 1>17-1-2-2-\mathrm{M}$ \\
\hline 5 & PCT - $4 \backslash \mathrm{SA} \backslash 1 \backslash 1, \mathrm{Bo} \backslash 6 \backslash 1>17-1-2-3-\mathrm{M}$ \\
\hline 6 & РCT - $4 \backslash \mathrm{SA} \backslash 1 \backslash 1, \mathrm{Bo} \backslash 6 \backslash 1>17-1-2-4-\mathrm{M}$ \\
\hline 7 & PCT - $4 \backslash \mathrm{SA} \backslash 1 \backslash 1, \mathrm{Bo} \backslash 6 \backslash 1>17-3-1-2-\mathrm{M}$ \\
\hline 8 & PCT - $4 \backslash \mathrm{SA} \backslash 1 \backslash 1, \mathrm{Bo} \backslash 6 \backslash 1>17-3-1-3-\mathrm{M}$ \\
\hline 9 & PCT - $4 \backslash \mathrm{SA} \backslash 1 \backslash 1, \mathrm{Bo} \backslash 6 \backslash 1>17-4-1-2-\mathrm{M}$ \\
\hline 10 & РCТ - $11 \backslash 0 \backslash 0 \backslash 2$, Bo $\backslash 1 \backslash 1>5-1-2-3-\mathrm{M}$ \\
\hline 11 & РCT - $11 \backslash 0 \backslash 0 \backslash 2$, , Во $\backslash 1 \backslash 1>5-1-3-1-\mathrm{M}$ \\
\hline 12 & PCT - $11 \backslash 0 \backslash 0 \backslash 2$, Bo $\backslash 1 \backslash 1>5-1-3-2-\mathrm{M}$ \\
\hline 13 & РСТ - $11 \backslash 0 \backslash 0 \backslash 2$, Bо $\backslash 1 \backslash 1>5-1-3-3-\mathrm{M}$ \\
\hline 14 & PCT $-11 \backslash 0 \backslash 0 \backslash 2, \mathrm{Bo} \quad \backslash 1 \backslash 1>17 \quad-2-3-2-\mathrm{M}$ \\
\hline 15 & РCT - $11 \backslash 0 \backslash 0 \backslash 2$, Bо $\backslash 1 \backslash 1>17 \quad-2-3-3-\mathrm{M}$ \\
\hline 16 & PCT - $11 \backslash 0 \backslash 0 \backslash 2$, Bо $\backslash 1 \backslash 1>17 \quad-3-1-3-\mathrm{M}$ \\
\hline 17 & PCT - $11 \backslash 0 \backslash 0 \backslash 2$, Bо $\backslash 1 \backslash 1>17 \quad-4-1-2-\mathrm{M}$ \\
\hline 18 & PCT - $11 \backslash 0 \backslash 0 \backslash 2$, Bо $\backslash 1 \backslash 1>17 \quad-5-4-1-\mathrm{M}$ \\
\hline 19 & PCT $-11 \backslash 0 \backslash 0 \backslash 2, \mathrm{Bo} \quad \backslash 1 \backslash 1>17 \quad-5-4-2-\mathrm{M}$ \\
\hline 20 & РCT - $11 \backslash 0 \backslash 0 \backslash 2$, Bо $\backslash 1 \backslash 1>17 \quad-5-4-3-\mathrm{M}$ \\
\hline 21 & PCT - $4 \backslash \mathrm{SA} \backslash 8 \backslash 1>19-1-1-2-1-\mathrm{M}$ \\
\hline 22 & 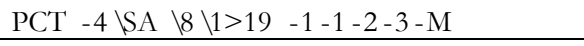 \\
\hline 23 & РCТ - $11 \backslash 0 \backslash 0 \backslash 2$, Bо $\backslash 4 \backslash 1>8-1-1-1-2-\mathrm{M}$ \\
\hline 24 & РCТ - $11 \backslash 0 \backslash 0 \backslash 2$, Bo $\backslash 4 \backslash 1>8-1-1-1-3-\mathrm{M}$ \\
\hline 25 & РCТ - $11 \backslash 0 \backslash 0 \backslash 2$, Bo $\backslash 4 \backslash 1>8-1-1-1-4-\mathrm{M}$ \\
\hline 26 & РCT - $11 \backslash 0 \backslash 0 \backslash 2$, Bo $\backslash 4 \backslash 1>8-1-1-1-6-\mathrm{M}$ \\
\hline 27 & РCT $-11 \backslash 0 \backslash 0 \backslash 2$, , Во $\backslash 4 \backslash 1>8-1-1-1-9-\mathrm{M}$ \\
\hline 28 & РCT - $11 \backslash 0 \backslash 0 \backslash 2$, Bо $\backslash 4 \backslash 1>8-1-2-2-2-\mathrm{M}$ \\
\hline 29 & РCT - $11 \backslash 0 \backslash 0 \backslash 2$, Bo $\backslash 4 \backslash 1>8-1-2-2-3-\mathrm{M}$ \\
\hline 30 & РCT - $11 \backslash 0 \backslash 0 \backslash 2$, Bo $\backslash 4 \backslash 1>8-1-2-2-6-\mathrm{M}$ \\
\hline 31 & РCТ - $11 \backslash 0 \backslash 0 \backslash 2$, , Во $\backslash 4 \backslash 1>8-1-2-8-1-\mathrm{M}$ \\
\hline 32 & PCT - $11 \backslash 0 \backslash 0 \backslash 2$, Bo $\backslash 4 \backslash 1>8-1-3-2-3-\mathrm{M}$ \\
\hline 33 & PCT $-11 \backslash 0 \backslash 0 \backslash 2$, Bo $\backslash 4 \backslash 1>8-1-3-4-1-\mathrm{M}$ \\
\hline 34 & РCT - $11 \backslash 0 \backslash 0 \backslash 2$, Bo $\backslash 4 \backslash 1>8-1-3-6-5-\mathrm{M}$ \\
\hline 35 & РCT $-11 \backslash 0 \backslash 0 \backslash 2$, Bо $\backslash 4 \backslash 1>8-1-4-2-1-\mathrm{M}$ \\
\hline 36 & РCТ - $11 \backslash 0 \backslash 0 \backslash 2$, Bо $\backslash 4 \backslash 1>8-1-4-2-4-\mathrm{M}$ \\
\hline 37 & РCТ - $11 \backslash 0 \backslash 0 \backslash 2, \mathrm{Bo} \quad \backslash 4 \backslash 1>8-1-4-8-2-\mathrm{M}$ \\
\hline 38 & РCT $-11 \backslash 0 \backslash 0 \backslash 2$, Bo $\backslash 4 \backslash 1>8-1-4-10-1-\mathrm{M}$ \\
\hline 39 & РCT - $11 \backslash 0 \backslash 0 \backslash 2$, Bo $\backslash 4 \backslash 1>8-1-8-5-1-\mathrm{M}$ \\
\hline 40 & РCT - $11 \backslash 0 \backslash 0 \backslash 2$, Bо $\backslash 4 \backslash 1>8-1-10-7-4-\mathrm{M}$ \\
\hline 41 & PCT - $11 \backslash 0 \backslash 0 \backslash 2$, Bo $\backslash 4 \backslash 1>8-1-10-8-1-\mathrm{M}$ \\
\hline 42 & PCT $-11 \backslash 0 \backslash 0 \backslash 2$, Bo $\backslash 4 \backslash 1>8-1-10-8-3-M$ \\
\hline 43 & PCT - $11 \backslash 0 \backslash 0 \backslash 2$, , Во $\backslash 4 \backslash 1>8-1-10-8-7-\mathrm{M}$ \\
\hline 44 & РCT $-11 \backslash 0 \backslash 0 \backslash 2$, Bо $\backslash 5 \backslash 1>56-2-1-3-6-\mathrm{M}$ \\
\hline 45 & PCT $-11 \backslash 0 \backslash 0 \backslash 2$, , $о$ o $\backslash 5 \backslash 1>80-3-4-2-2-M$ \\
\hline 46 & РСТ - $11 \backslash 0 \backslash 0 \backslash 2$, Bо $\backslash 5 \backslash 1>80-3-4-2-3-\mathrm{M}$ \\
\hline 47 & РCТ $-11 \backslash 0 \backslash 0 \backslash 2$, Bо $\backslash 5 \backslash 1>102 \quad-2-1-3-3-\mathrm{M}$ \\
\hline 48 & PCT $-11 \backslash 0 \backslash 0 \backslash 2$, Bо $\backslash 5 \backslash 1>102 \quad-2-1-3-4-\mathrm{M}$ \\
\hline 49 & РCТ - $11 \backslash 0 \backslash 0 \backslash 2$, Во $\backslash 5 \backslash 1>92-1-5-6-3-\mathrm{M}$ \\
\hline 50 & PCT - $11 \backslash 0 \backslash 0 \backslash 2$, , Во $\backslash 5 \backslash 1>92-3-2-2-2-\mathrm{M}$ \\
\hline 51 (T.) & CORPOICA Llanura 11, Línea 30 o CIRAD 409 \\
\hline
\end{tabular}


Tabla 2. Media cuadrática para las variables Pyricularia en Hojas -PHOJAS-; Pyricularia en cuello de panículas -PC-; manchado de granos -MGRA-; Helminthosporiosis -HELM-; escaldado en hojas -ESC-; vigor vegetativo -VIGOR-; días a floración -DFLO- y rendimiento -REND-, en 51 líneas de arroz, evaluadas en un suelo de sabana ácida. Villavicencio, 2017B.

\begin{tabular}{|l|c|c|c|c|c|c|c|c|c|}
\hline $\begin{array}{c}\text { Fuente de } \\
\text { variación }\end{array}$ & $\begin{array}{c}\text { Grados } \\
\text { de } \\
\text { libertad }\end{array}$ & PHOJAS & PC & MGRA & HELM & ESC & VIGOR & DFLO & REND \\
\hline Bloques & 2 & 0,55 & 2,38 & 3,24 & $21,36^{* *}$ & 0,12 & $11,61^{* *}$ & $92,20^{*}$ & $4679344,99^{*}$ \\
\hline Líneas & 50 & $0,68^{*}$ & 0,87 & $5,90^{* *}$ & 0,75 & 1,00 & $3,24^{* *}$ & $159,29^{* *}$ & $10584023,91^{* *}$ \\
\hline Error & 100 & 0,36 & 1,02 & 1,44 & 0,72 & 0,91 & 1,05 & 11,29 & 1186570,53 \\
\hline Total & 152 & & & & & & & & \\
\hline
\end{tabular}

*Significativo al nivel del $5 \% \quad * *$ Significativo al nivel del $1 \%$

Respecto de la evaluación de las enfermedades más limitantes en la zona, en cuanto a Pyricularia en hojas, se presentó diferencia significativa entre líneas (Tabla 2). Todas las seis líneas de mejor desempeño en rendimiento de grano de la población PCT-11 y la línea 22 de PCT-4, presentaron incidencias bajas de Pyricularia en hojas y menores al testigo comercial Corpoica Llanura 11 (Tabla 3), el cual, por haberse sembrado comercialmente desde hace más de diez años, presenta una mayor incidencia de dicho patógeno.

En cuanto a la incidencia de manchado de grano y el vigor vegetativo, las líneas de mayores rendimientos presentaron buen comportamiento y sin diferencia significativa con el testigo comercial. Por el contrario, las 9 líneas más tardías de la población PCT-4 presentaron bajo vigor vegetativo y alta incidencia de manchado de grano (Tabla 3), favorecido por alta esterilidad de los mismos.

Respecto a la precocidad de las líneas, el testigo comercial Corpoica Llanura 11, presentó la mayor precocidad con 60 días a floración (Tabla 3). Ninguna de las líneas en evaluación igualó esta destacada característica, que favorece la siembra de otro cultivo de rotación, durante el año agrícola (Delgado \& Aristizábal, 2002). Las líneas de la población PCT-11, con mayor rendimiento de grano de las evaluadas, presentaron floraciones cercanas a los 65 días (Tabla 3), siendo también precoces y de adecuado rendimiento.

La línea 47 que visualmente presentó buen fenotipo de planta en estado vegetativo y baja incidencia de enfermedades, obtuvo un rendimiento de $5.009 \mathrm{~kg} \mathrm{ha}^{-1}$, sin diferencia significativa de las líneas de mayor rendimiento y presentó buena precocidad y vigor (Tabla 3).

En trigo, la SR fue usada exitosamente para la obtención de genotipos excepcionalmente vigorosos, con altas variaciones genéticas y heredabilidades para el ancho y el área total de la hoja en cada ciclo (Zhang et al. 2015).

Morais Júnior et al. (2017a), utilizaron la población de arroz de riego genéticamente amplia, CNA12S, en tres ciclos de selección, manteniéndose la variabilidad genética durante los ciclos. La ganancia genética promedio por año fue de 1,98\%, para el rendimiento de grano y $-1,29 \%$, para días a floración y se aumentó el potencial genético de la población, para la extracción de líneas superiores, mostrando la efectividad del método de SR, en el mejoramiento de arroz de riego.

De forma similar en Brasil, después de cuatro ciclos de SR, en la población CNA6 de arroz de tierras altas, aumentaron efectiva-mente el potencial genético de la población, para generar líneas endogámicas superiores, para rendimiento de grano y altura de planta, manteniendo el potencial genético para los días a floración y mostrando la efectividad de la selección recurrente en arroz de secano en sabanas ácidas (Morais Júnior et al. 2015; Morais Júnior et al. 2017b).

También, Herawati et al. (2017b) evaluaron y seleccionaron por SR, el comportamiento a estrés por sequía, de 180 líneas de arroz de tierras altas en estado de plántula, mostrando 53 líneas tolerantes, 99 moderadamente tolerantes y 28 susceptibles, comprobando la efectividad de la SR, para el mejoramiento de la tolerancia a sequía en arroz de sabanas.

Los resultados del presente trabajo muestran la utilidad de la SR para generar nuevas líneas superiores en rendimiento de grano, resisten-cia a enfermedades, adaptación y para contribuir a la diversificación de la base genética del arroz en las sabanas ácidas de los Llanos. Las líneas seleccionadas y otras provenientes del mismo programa de SR, se deberán seguir evaluando en sabanas de la altillanura pla-na, para la futura obtención de nuevas variedades mejoradas, con mayor estabilidad en su comportamiento agronómico, gracias a su mayor variabilidad genética incorporada.

Conflictos de intereses: El manuscrito fue preparado y revisado con la participación de todos los autores, quienes declaramos que no existe ningún conflicto de intereses que ponga en riesgo la vali-dez de los resultados presentados. Financiación: Esta investigación fue financiada por la Dirección General de Investigaciones de la Universidad de los Llanos, Código C05F01-027-2016. 
Tabla 3. Diferencias de medias (P. de Tukey), para las variables con diferencia significativa entre líneas en el Anava. 2017 B.

\begin{tabular}{|c|c|c|c|c|c|}
\hline LÍNEA & RENDIMIENTO & PHOJAS & MGRA & VIGOR & DFLO \\
\hline 39 & $6470,84 \mathrm{~A}$ & $1,00 \mathrm{~B}$ & $1,67 \mathrm{CD}$ & $2,33 \mathrm{AB}$ & $65,67 \mathrm{CDE}$ \\
\hline 37 & $6192,27 \mathrm{~A}$ & $1,67 \mathrm{AB}$ & 2,33 BCD & $3,00 \mathrm{AB}$ & $64,67 \mathrm{DE}$ \\
\hline 34 & $6178,16 \mathrm{AB}$ & $1,67 \mathrm{AB}$ & $2,33 \mathrm{BCD}$ & $3,00 \quad \mathrm{AB}$ & $66,00 \mathrm{CDE}$ \\
\hline 48 & $6092,67 \mathrm{AB}$ & $1,00 \mathrm{~B}$ & $2,33 \mathrm{BCD}$ & $3,00 \mathrm{AB}$ & $66,00 \mathrm{CDE}$ \\
\hline 36 & $6077,63 \mathrm{AB}$ & $1,00 \mathrm{~B}$ & $2,33 \mathrm{BCD}$ & $3,00 \mathrm{AB}$ & $64,67 \mathrm{DE}$ \\
\hline 23 & $6071,95 \mathrm{AB}$ & $1,00 \mathrm{~B}$ & $1,67 \mathrm{CD}$ & $4,33 \mathrm{AB}$ & $65,00 \mathrm{DE}$ \\
\hline 32 & $5817,83 \mathrm{AB}$ & $1,00 \mathrm{~B}$ & $1,67 \mathrm{CD}$ & $3,00 \mathrm{AB}$ & $65,33 \mathrm{DE}$ \\
\hline 29 & $5620,58 \mathrm{AB}$ & $1,67 \mathrm{AB}$ & $3,00 \mathrm{ABCD}$ & $3,67 \mathrm{AB}$ & $66,00 \mathrm{CDE}$ \\
\hline 25 & $5403,58 \mathrm{AB}$ & $1,67 \mathrm{AB}$ & $2,33 \mathrm{BCD}$ & $4,33 \mathrm{AB}$ & $64,67 \mathrm{DE}$ \\
\hline 33 & $5315,87 \mathrm{AB}$ & $1,00 \mathrm{~B}$ & 1,67 CD & $3,00 \mathrm{AB}$ & $67,00 \mathrm{CDE}$ \\
\hline 26 & $5280,05 \mathrm{AB}$ & $1,00 \mathrm{~B}$ & $3,00 \mathrm{ABCD}$ & $3,67 \mathrm{AB}$ & $68,00 \mathrm{CDE}$ \\
\hline 45 & $5265,67 \mathrm{AB}$ & $1,00 \mathrm{~B}$ & $2,33 \mathrm{BCD}$ & $2,33 \mathrm{AB}$ & $64,67 \mathrm{DE}$ \\
\hline 24 & $5137,70 \mathrm{AB}$ & $1,00 \mathrm{~B}$ & $3,00 \mathrm{ABCD}$ & $3,67 \mathrm{AB}$ & $65,33 \mathrm{DE}$ \\
\hline 30 & $5118,09 \quad \mathrm{AB}$ & $1,67 \mathrm{AB}$ & $3,00 \mathrm{ABCD}$ & $3,67 \mathrm{AB}$ & 68,67 CDE \\
\hline 28 & $5117,70 \mathrm{AB}$ & $1,00 \mathrm{~B}$ & $1,67 \mathrm{CD}$ & $3,00 \mathrm{AB}$ & $70,00 \mathrm{CDE}$ \\
\hline 12 & $5071,75 \mathrm{AB}$ & $1,00 \mathrm{~B}$ & $3,67 \mathrm{ABCD}$ & $1,67 \mathrm{~B}$ & $66,33 \mathrm{CDE}$ \\
\hline 47 & $5008,88 \mathrm{AB}$ & $1,00 \mathrm{~B}$ & $1,00 \mathrm{D}$ & $2,33 \mathrm{AB}$ & $63,67 \mathrm{DE}$ \\
\hline 35 & $4980,71 \mathrm{AB}$ & $1,00 \mathrm{~B}$ & 1,67 CD & $3,67 \mathrm{AB}$ & $66,00 \mathrm{CDE}$ \\
\hline 50 & $4973,91 \mathrm{AB}$ & $1,00 \mathrm{~B}$ & 2,33 BCD & $2,33 \mathrm{AB}$ & $67,00 \mathrm{CDE}$ \\
\hline 43 & $4933,00 \mathrm{AB}$ & $1,67 \mathrm{AB}$ & $3,00 \mathrm{ABCD}$ & $3,00 \mathrm{AB}$ & $66,67 \mathrm{CDE}$ \\
\hline 31 & $4771,88 \mathrm{AB}$ & $1,00 \mathrm{~B}$ & $1,67 \mathrm{CD}$ & $3,00 \mathrm{AB}$ & 66,33 CDE \\
\hline 22 & $4751,04 \mathrm{AB}$ & $1,67 \mathrm{AB}$ & $1,67 \mathrm{CD}$ & $2,33 \mathrm{AB}$ & $71,67 \mathrm{BCD}$ \\
\hline 27 & $4713,72 \mathrm{AB}$ & $1,67 \mathrm{AB}$ & $2,33 \mathrm{BCD}$ & $3,67 \mathrm{AB}$ & $68,33 \mathrm{CDE}$ \\
\hline 40 & $4684,63 \mathrm{AB}$ & $1,67 \mathrm{AB}$ & $3,00 \mathrm{ABCD}$ & $3,67 \mathrm{AB}$ & $64,33 \mathrm{DE}$ \\
\hline 38 & $4631,43 \mathrm{AB}$ & $2,33 \mathrm{AB}$ & $2,33 \mathrm{BCD}$ & $3,67 \mathrm{AB}$ & 69,00 CDE \\
\hline 41 & $4564,96 \mathrm{AB}$ & $1,00 \mathrm{~B}$ & $2,33 \mathrm{BCD}$ & $3,00 \mathrm{AB}$ & $67,67 \mathrm{CDE}$ \\
\hline 46 & $4487,31 \mathrm{AB}$ & $1,00 \mathrm{~B}$ & $3,00 \mathrm{ABCD}$ & $2,33 \mathrm{AB}$ & $64,67 \mathrm{DE}$ \\
\hline 44 & $4472,80 \mathrm{AB}$ & $1,00 \mathrm{~B}$ & $2,33 \mathrm{BCD}$ & $3,67 \mathrm{AB}$ & 69,00 CDE \\
\hline 42 & $4369,27 \mathrm{ABC}$ & $1,00 \mathrm{~B}$ & $3,00 \mathrm{ABCD}$ & $3,67 \mathrm{AB}$ & 69,00 CDE \\
\hline 15 & $4115,29 \mathrm{ABCD}$ & $1,00 \mathrm{~B}$ & $1,67 \mathrm{CD}$ & $3,00 \mathrm{AB}$ & 69,00 CDE \\
\hline 49 & 4104,11 ABCDE & $1,00 \mathrm{~B}$ & $1,67 \mathrm{CD}$ & $3,00 \mathrm{AB}$ & 66,00 CDE \\
\hline 17 & 3960,71 ABCDEF & $1,00 \mathrm{~B}$ & $1,67 \mathrm{CD}$ & $3,00 \mathrm{AB}$ & $67,00 \mathrm{CDE}$ \\
\hline 11 & 3797,25 ABCDEFG & $1,00 \mathrm{~B}$ & $1,67 \mathrm{CD}$ & $1,67 \mathrm{~B}$ & $68,33 \mathrm{CDE}$ \\
\hline 18 & 3548,49 ABCDEFG & $1,00 \mathrm{~B}$ & $1,67 \mathrm{CD}$ & $2,33 \quad \mathrm{AB}$ & $65,67 \mathrm{CDE}$ \\
\hline 19 & 3523,33 ABCDEFG & $1,00 \mathrm{~B}$ & $2,33 \mathrm{BCD}$ & $3,67 \mathrm{AB}$ & $66,33 \mathrm{CDE}$ \\
\hline 13 & 3413,39 ABCDEFG & $1,00 \mathrm{~B}$ & $2,33 \mathrm{BCD}$ & $3,67 \mathrm{AB}$ & 64,67 DE \\
\hline 20 & 3252,28 ABCDEFG & $1,00 \mathrm{~B}$ & $2,33 \mathrm{BCD}$ & $3,67 \mathrm{AB}$ & $68,33 \mathrm{CDE}$ \\
\hline 16 & 3183,91 ABCDEFG & $1,67 \mathrm{AB}$ & $1,00 \mathrm{D}$ & $5,00 \mathrm{AB}$ & $70,00 \mathrm{CDE}$ \\
\hline 14 & 3159,67 ABCDEFG & $1,67 \mathrm{AB}$ & $3,00 \mathrm{ABCD}$ & $3,00 \mathrm{AB}$ & $67,67 \mathrm{CDE}$ \\
\hline 10 & 3158,49 ABCDEFG & $1,00 \mathrm{~B}$ & $3,00 \mathrm{ABCD}$ & $3,67 \mathrm{AB}$ & $72,00 \mathrm{BCD}$ \\
\hline 21 & 2967,58 ABCDEFG & $1,67 \mathrm{AB}$ & $3,00 \mathrm{ABCD}$ & $3,00 \mathrm{AB}$ & $72,00 \mathrm{BCD}$ \\
\hline 51 (T.) & 2510,19 BCDEFG & $3,67 \mathrm{~A}$ & $1,67 \mathrm{CD}$ & $5,00 \mathrm{AB}$ & $59,67 \mathrm{E}$ \\
\hline 07 & $754,97 \quad$ CDFG & $1,00 \mathrm{~B}$ & $6,33 \mathrm{AB}$ & $5,00 \mathrm{AB}$ & $83,67 \mathrm{~A}$ \\
\hline 06 & 635,82 DEFG & $1,00 \mathrm{~B}$ & $6,67 \mathrm{~A}$ & $5,00 \mathrm{AB}$ & $85,67 \mathrm{~A}$ \\
\hline 08 & $430,98 \quad$ EFG & $1,00 \mathrm{~B}$ & $5,67 \mathrm{ABC}$ & $5,67 \mathrm{~A}$ & $85,00 \mathrm{~A}$ \\
\hline 04 & $383,01 \quad F G$ & $1,00 \mathrm{~B}$ & $5,00 \mathrm{ABCD}$ & $5,00 \mathrm{AB}$ & $86,00 \mathrm{AB}$ \\
\hline 09 & $344,64 \quad F G$ & $1,00 \mathrm{~B}$ & $5,67 \mathrm{ABC}$ & $5,67 \mathrm{~A}$ & $87,67 \mathrm{~A}$ \\
\hline 03 & $307,25 \quad F G$ & $1,00 \mathrm{~B}$ & $5,00 \mathrm{ABCD}$ & $3,67 \mathrm{AB}$ & $85,67 \mathrm{ABC}$ \\
\hline 02 & $291,57 \quad F G$ & $1,00 \mathrm{~B}$ & $5,33 \mathrm{ABC}$ & $5,00 \mathrm{AB}$ & 83,00 ABCDE \\
\hline 01 & $277,32 \quad G$ & $1,00 \mathrm{~B}$ & $5,00 \mathrm{ABCD}$ & $5,67 \mathrm{~A}$ & 77,00 ABCDEF \\
\hline 05 & $263,01 \quad G$ & $1,00 \mathrm{~B}$ & 5,00 ABCD & $5,67 \mathrm{~A}$ & $87,67 \mathrm{~A}$ \\
\hline
\end{tabular}




\section{REFERENCIAS}

1. ABDULLAH, B. 2009. Progress of rice improvement through recurrent selection. J. Agron. Indonesia. 37(3):188-193.

2. ANSARI, A.; WANG, C.; WANG, J.; WANG, F.; LIU, P.; GAO, Y.; TANG, Y.; ZHAO, K. 2017. Engineered dwarf malesterile rice: A promising genetic tool for facilitating recurrent selection in rice. Front. Plant Sci. 8(2132):1-11. https://doi.org/10.3389/fpls.2017.02132

3. BERILLI, A.P.C.G.; PEREIRA, M.G.; TRINDADE, R.D.S.; COSTA, F.R.D.; CUNHA, K.S.D. 2013. Response to the selection in the 11 th cycle of reciprocal recurrent selection among full-sib families of maize. Acta Scientiarum, Agron. $35: 435-441$.

https.//doi.org/10.4025/actasciagron.v35i4.17489

4. CHATEL, M.; GUIMARAES, E.P. 2000. Catalogue registration to manage rice gene pools and population improvement. Proyecto CIRAD/CIAT. Centre de coopèration Internationale en Recherche Agronomique pour le Dèveloppement-Deparment des cultures annuelles (CIRAD-CA) y Centro Internacional de Agricultura Tropical (CIAT), Cali, Colombia. 75p.

5. CHATEL, M.; GUIMARAES, E.P.; OSPINA, Y.; RODRIGUEZ, F.; LOZANO, V.H. 2010. Mejoramiento de poblaciones de arroz de secano empleando selección recurrente y desarrollo de variedades. En: Degiovani, V.; Martinez, C.P.; Motta, F. (eds). Producción Eco-Eficiente del Arroz en América Latina. (Tomo I, capítulos 1-24.) Publicación CIAT, Cali. $\mathrm{N}^{\circ}$ 365. $487 \mathrm{p}$.

6. CHATEL, M.; OSPINA, Y.; RODRIGUEZ, F.; LOZANO, V.H.; DELGADO, H. 2008. Upland rice composite population breeding and selection of promising lines for colombian savannah ecosystem. Pesquisa Agropecuaria Tropical. 38(1):1-5.

7. CUEVAS-PEREZ, F.E.; GUIMARAES, E.P.; BERRÍO, L.E.; GONZALES, D.I. 1992. Genetic base of irrigated rice in Latin America and the Caribbean, 1971 to 1989. Crop Science. 32:1054-1059.

https://doi.org/10.2135/cropsci1992.0011183X00320004 $0044 x$

8. DAS, S.R. 2018. Conventional breeding approaches for enhancing yield potential of rice. Oryza. 55(Special Issue):1-17. https://doi.org/10.5958/2249-5266.2018.00001.2

9. DELGADO, H.; ARISTIZÁBAL, D. 2002. Potencialidad de una línea promisoria de arroz de alta precocidad para suelos ácidos de los Llanos Orientales. Fitotecnia Colombiana. $2(1): 47-59$.
10. DELGADO, H.; CHATEL, M.; OSPINA, Y. 2004. Llanura 11, nueva variedad de arroz para el ecosistema de sabana en la altillanura colombiana. Fitotecnia Colombiana. 4(2):8-11.

11. GAZAL, A.; DAR, Z.A.; WANI, S.H.; LONE, A.A.; SHIKARI, A.B.; ALI, G.; ABIDI, I. 2016. Molecular breeding for enhancing resilience against biotic and abiotic stress in major cereals. SABRAO J. Breeding and Genetics. 48(1):1-32.

12. GRENIER, C.; CAO, T.V.; OSPINA, Y.; QUINTERO, C.; CHATEL, M.H.; TOHME, J.; COURTOIS, B.; AHMADI, N. 2015. Accuracy of genomic selection in a rice synthetic population developed for recurrent selection breeding. PLOS ONE. 10(8):1-25.

https://doi.org/10.1371/journal.pone.0136594

13. HALlAUER, A.R.; FILHO, J.B.M.; CARENA, M.J. 2010. Germplasm. In: Quantitative Genetics in Maize Breeding. Handbook of Plant Breeding, vol 6. Springer, New York, NY.

14. HERAWATI, R.; INORIAH, E.; RUSTIKAWATI; MUKHTASAR. 2017a. Genetics diversity and characters agronomic of F3 lines selected by recurrent selection for drought tolerance and blast resistance of Bengkulu local rice varieties. IJASEIT. 7(3):922-927.

https://doi.org/10.18517/ijaseit.7.3.1641

15. HERAWATI, R.; MASDAR; GANEFIANTI, D.Y; HERMAWAN, B.; ALNOPRI. 2017b. Screening and identification of upland rice lines derived recurrent selection for drought tolerance. IJASEIT. 7(6):2322-2327. https://doi.org/10.18517/ijaseit.7.6.2955

16. MONTALVAN, R.; DESTRO, D.; DA SILVA E.F.; MONTAÑO, J.C. 1998. Genetic base of Brazilian upland rice cultivars. J. Genetic Breeding. 52:203-209.

17. MORAIS JÚNIOR, O.P.; MELO, P.G.S.; MORAIS, O.P.; CASTRO, O.P.; BRESEGHELLO, F.; UTUMI, M.M.; PEREIRA, J.A.; WRUCK, F.J.; FILHO, J.M.C. 2015. Genetic progress after cycles of upland rice recurrent selection. Sci. Agric. 72(4):297-305. http://dx.doi.org/10.1590/0103-9016-2014-0137

18. MORAIS JÚNIOR, O.P.; BRESEGHELLO, F; DUARTE, J.B.; MORAIS, O.P.; RANGEL, P.H.N.; COELHO, A.S.G. 2017a. Effectiveness of recurrent selection in irrigated rice breeding. Crop Science. 57(6):3043-3058. https://doi.org/10.2135/cropsci2017.05.0276

19. MORAIS JÚNIOR, O.P.; MELO, P.G.S.; MORAIS, O.P.; FILHO, J.M.C. 2017b. Genetic variability during four cycles of recurrent selection in rice. Pesquisa Agropecuária Brasileira. 52(11):1033-1041. https://doi.org/10.1590/S0100-204X2017001100009 
20. PANG, Y.; CHEN, K.; WANG, X.; XU, J.; ALI, J.; LI, Z. 2017. Recurrent selection breeding by dominant male sterility for multiple abiotic stresses tolerant rice cultivars. Euphytica. 213(article 268):13.

https://doi.org/10.1007/s10681-017-2055-5

21. RANGEL, P.H.N.; GUIMARAES, E.P.; NEVES, P. DE C.F. 1996. Base genética das cultivares de arroz (Oryza sativa L.) irrigado do Brasil. Pesquisa Agropecuaria Brasileira. 31(5):349-357.

22. ROSERO, M. 1983. Sistema de Evaluación Estandar para Arroz. Segunda Edición. Centro Internacional De Agricultura Tropical, CIAT. Cali, Colombia. 61p.

23. SINGH, R.J.; IKEHASHI, H.I. 1981. Monogenic male-sterility in rice: Introduction, identification and inheritance. Crop Scince. 21:286-289.

https://doi.org/10.2135/CROPSCI1981.0011183X002100020020X
24. XU, J.L.; ALI, J. 2014. Connecting rice germplasm to plant breeding: Backcrossing for allele mining and recurrent selection for allele pyramiding through molecular marker technology. Adv. Crop Sci. Tech. 2:e114. https://doi.org/10.4172/2329-8863.1000e114

25. ZHANG, L.; RICHARDS, R.A.; CONDON, A.G.; LIU, D.C.; REBETZKE, G.J. 2015. Recurrent selection for wider seedling leaves increases early biomass and leaf area in wheat (Triticum aestivum L.). J. Experimental Botany. 66(5):1215-1226.

https://doi.org/10.1093/jxb/eru468 